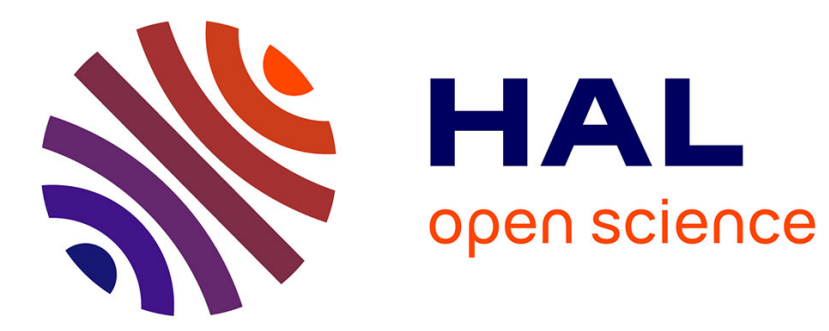

\title{
Frequency Design of Interconnected Dissipative Systems: a Unified LMI Approach
}

Arthur Perodou, Anton Korniienko, Mykhailo Zarudniev, Gérard Scorletti

\section{To cite this version:}

Arthur Perodou, Anton Korniienko, Mykhailo Zarudniev, Gérard Scorletti. Frequency Design of Interconnected Dissipative Systems: a Unified LMI Approach. CDC, Dec 2018, Miami, FL, United States. 10.1109/CDC.2018.8618932 . hal-01984416

\section{HAL Id: hal-01984416 https://hal.science/hal-01984416}

Submitted on 17 Jan 2019

HAL is a multi-disciplinary open access archive for the deposit and dissemination of scientific research documents, whether they are published or not. The documents may come from teaching and research institutions in France or abroad, or from public or private research centers.
L'archive ouverte pluridisciplinaire HAL, est destinée au dépôt et à la diffusion de documents scientifiques de niveau recherche, publiés ou non, émanant des établissements d'enseignement et de recherche français ou étrangers, des laboratoires publics ou privés. 


\title{
Frequency Design of Interconnected Dissipative Systems: a Unified LMI Approach*
}

\author{
Arthur Perodou ${ }^{1,2}$, Anton Korniienko ${ }^{2}$, Mykhailo Zarudniev $^{3}$ and Gérard Scorletti ${ }^{2}$
}

\begin{abstract}
This paper deals with the design of a system, defined as the interconnection of identical LTI subsystems, whose frequency-response is under modulus constraints. Based on the notions of LFT and dissipativity, we propose a method able to compute a solution by solving a linear minimisation problem under LMI constraints. This extends the usual approach by especially generalising the spectral factorisation technique from systems with state-space model to identical dissipative systems interconnection.
\end{abstract}

Keywords: Filter Design, LFT/LFR systems, Spectral Factorisation, LMI, Dissipativity

\section{INTRODUCTION}

Modern engineering systems have become increasingly complex. Not only systems have become complex, but also the way they are interconnected in, leading to challenging engineering problems. In the particular case where interconnected systems are identical, a new paradigm has emerged, known as large-scale (distributed/decentralised) systems or homogeneous multi-agent systems approach. In the last decade, this approach has been successful in diverse areas of application such as formation flying [1], micro-Electronics [2], [3], biological networks [4], etc.

These systems have the common property to be described by a Linear Fractional Representation (LFR). The LFR is the generalisation of the state-space representation. Homogeneous LTI LFR systems are defined as the feedback interconnection between a gain matrix with identical Linear Time-Invariant (LTI) subsystems $T_{s}$. While the state-space approach can efficiently solve many standard problems, using especially Linear Matrix Inequalities (LMIs) [5], it appears to be more limited when considering interconnected complex systems. Many useful state-space results have then been extended to homogeneous LTI LFR systems [2], [4], [6], [7]. Nevertheless, there are still extended standard problems to solve. One of them is the computation of the transfer function of an homogeneous LTI LFR system, whose frequencyresponse is under modulus constraints. It is a generalisation of a generic problem with important applications in Signal Processing (frequency filter design) and Automatic Control

*The authors gratefully acknowledge the financial supports from Région Rhône-Alpes (ARC6), Ecole Doctorale EEA de Lyon and IEEE Control Systems Society (STA).

${ }^{1}$ A. Perodou is with Institute of Nanotechnology of Lyon (INL), Ecole Centrale de Lyon, Université de Lyon, 69130 Ecully, France. arthur. perodouddoctorant.ec-lyon. fr

${ }^{2}$ A. Perodou, A. Korniienko and G. Scorletti are with Laboratoire Ampère, Ecole Centrale de Lyon, Université de Lyon, 69130 Ecully, France. \{anton.korniienko, gerard.scorletti\}@ec-lyon.fr

${ }^{3} \mathrm{M}$. Zarudniev is with CEA-TECH, MINATEC Campus, 38054 Grenoble, France. mykhailo.zarudniev@cea.fr
( $H_{\infty}$-weight computation) [8]. This is the main purpose of this paper.

To achieve this, we develop a new approach based on LFR and the dissipativity characterisation of the subsystems $T_{s}$. This extends the usual approach established for state-space systems, which consists of two steps, both relying on convex optimisation. First, a magnitude function $M(\omega)$ satisfying the modulus constraints is calculated. Then, a transfer function is computed from $M$, using the spectral factorisation technique.

Our first contribution is to extend the usual approach to any lossless dissipative $T_{s}$. For that purpose, the spectral factorisation technique is generalised to dissipative systems. This provides our second contribution. Finally, the lossy part of general dissipative systems introduces a new term which prevents from extending each step independently. Our third contribution is then to provide an alternative approach, still relying on convex optimisation, which reveals how to handle this term.

The paper is organised as follows: Section II introduces the notions of LFR and dissipativity. Two design problems of interconnected dissipative systems are defined in Section III, and are solved in Section IV. An illustrative example is given in Section V. Section VI concludes the paper.

We use the following notation. Lower (upper) case letters are used for vectors (matrices). $I_{n}$ denotes the identity matrix of $\mathbb{R}^{n \times n}$ and $0_{n \times m}$ the zero matrix of $\mathbb{R}^{n \times m}$. The subscripts are omitted when obvious from the context. $X^{T}$ and $X^{*}$ stand for transpose and transpose conjugate of $X$ respectively while $M>(\geq) 0$ denotes positive (semi-) definiteness. * represents the Redheffer star product [9]. In an optimisation problem, bold characters are, or depend on, decision variables. For sake of briefness, the additional notations are used:

$$
\begin{aligned}
& \mathcal{R}\left(\left[\begin{array}{ll}
A & B \\
C & D
\end{array}\right], P, \mathcal{X}\right)=A^{T}(-P)+(-P) A+C^{T} \mathcal{X} C \\
& -\left((-P) B+C^{T} \mathcal{X} D\right)\left(D^{T} \mathcal{X} D\right)^{-1}\left((-P) B+C^{T} \mathcal{X} D\right)^{T} \\
& \mathcal{L} \mathcal{M}\left(\left[\begin{array}{cc}
A & B \\
C & D
\end{array}\right], \mathcal{Y}, \mathcal{X}\right)=\left[\begin{array}{cc}
A & B \\
I & 0 \\
\hline C & D
\end{array}\right]^{T}\left[\begin{array}{c|c}
\mathcal{Y} & 0 \\
\hline 0 & \mathcal{X}
\end{array}\right]\left[\begin{array}{cc}
A & B \\
I & 0 \\
\hline C & D
\end{array}\right]
\end{aligned}
$$

\section{Preliminaries}

In this paper, systems are described using the LFR/LFT framework [9].

Definition $1(L F R / L F T)$ : A Linear Fractional Representa- 
tion (LFR) of a system $(G, \Delta)$ is a set of equations

$$
\left\{\begin{array}{c}
p=c \\
{\left[\begin{array}{l}
q \\
z
\end{array}\right]=\underbrace{\left[\begin{array}{l|l}
A & B \\
\hline C & D
\end{array}\right]}_{:=G}\left[\begin{array}{c}
p \\
w
\end{array}\right]}
\end{array}\right.
$$

where $G$ is a real matrix and $\Delta$ an operator. Provided that $(I-A \Delta)^{-1}$ exists, the Linear Fractional Transformation (LFT) is defined as $\Delta \star G:=D+C \Delta(I-A \Delta)^{-1} B$, and links the input $w$ and the output $z: z=(\Delta \star G) w$.

An important concept is the dissipativity characterisation of systems [10]. A specific version is used in the paper [3].

Definition 2 ( $\{X, Y, Z\}$-Dissipativity): Let $X=X^{T}, Y$, $Z=Z^{T}$ be real matrices and $\mathcal{I}$ be either $\mathbb{R}$ or a sub-interval of $\mathbb{R}$. An LTI system $T$ is $\{X, Y, Z\}$-dissipative on $\mathcal{I}$, if $\left[\begin{array}{cc}X & Y \\ Y^{T} & Z\end{array}\right]$ is full rank, $X \leq 0$ and

$$
\forall \omega \in \mathcal{I}, \quad\left[\begin{array}{c}
T(j \omega) \\
I
\end{array}\right]^{*}\left[\begin{array}{cc}
X & Y \\
Y^{T} & Z
\end{array}\right]\left[\begin{array}{c}
T(j \omega) \\
I
\end{array}\right] \geq 0
$$

If $\geq$ is replaced with $=$ in (2), the system will be said to be lossless $\{X, Y, Z\}$-dissipative on $\mathcal{I}$.

When $\mathcal{I}$ is not specified, it is assumed that $\mathcal{I}=\mathbb{R}$.

\section{Problems Statement}

In this section, we first define systems under consideration. The design problems are then presented.

\section{A. Identical Dissipative Systems Interconnection}

We focus on systems defined as the interconnection between a constant matrix and identical, SISO, LTI, $\left\{x_{d}, y_{d}, z_{d}\right\}$-dissipative subsystems $T_{s}$, and denoted $W\left(T_{s}\right)$. They are referred to as identical dissipative systems interconnection in the sequel.

Such systems can be represented by an LFR (1), where $\Delta=T_{s} \cdot I_{n}$. A compact formulation is then obtained:

$$
W\left(T_{s}\right)=\left(T_{s} \cdot I_{n}\right) \star\left[\begin{array}{c|c}
A_{W} & B_{W} \\
\hline C_{W} & D_{W}
\end{array}\right]
$$

and is represented by the block diagram of Fig. 1. Such

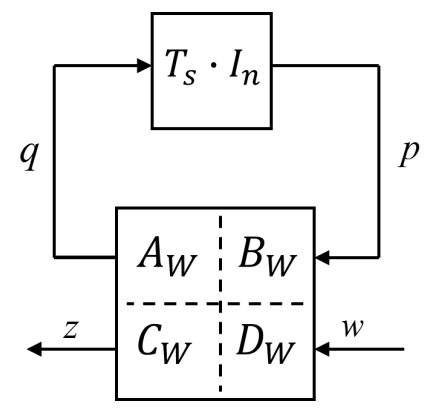

Fig. 1. Block Diagram of an identical dissipative systems interconnection

formulation is a generalisation of traditional LTI representations. When $T_{s}(s)=1 / s$ (which is lossless $\{0,1,0\}$ dissipative), the LFR corresponds to the continuous-time state-space representation.
The design problem is, given $T_{s}$, to compute the matrices $A_{W}, B_{W}, C_{W}$ and $D_{W}$ such that the frequency response $W\left(T_{s}(j \omega)\right)$ satisfies modulus constraints, called a spectral mask. A spectral mask is defined on frequency intervals (continuous-mask) or at frequency points (discrete-mask).

\section{B. Continuous-Mask Design Problem}

\section{Problem 1 (Continuous-Mask Formulation): Given}

1) $N_{U}$ positive real numbers $U_{i}, N_{U}$ intervals $\Omega_{U}^{i} \subseteq \mathbb{R}$

2) $N_{L}$ positive real numbers $L_{l}, N_{L}$ intervals $\Omega_{L}^{l} \subseteq \mathbb{R}$

Find if there exists a stable identical dissipative systems interconnection $W\left(T_{s}\right)$, such that:

$$
\begin{array}{lll}
\forall i \in\left\{1, \ldots, N_{U}\right\}, & \forall \omega \in \Omega_{U}^{i}, & \left|W\left(T_{s}(j \omega)\right)\right|^{2}<U_{i}^{2} \\
\forall l \in\left\{1, \ldots, N_{L}\right\}, & \forall \omega \in \Omega_{L}^{l}, & \left|W\left(T_{s}(j \omega)\right)\right|^{2}>L_{l}^{2}
\end{array}
$$

and compute it.

Remark 1: This problem is actually the extension of the usual frequency filter design [8], where $T_{s}(s)=1 / s$, to identical dissipative systems interconnection.

Remark 2: Conditions (4) and (5) extend to MultipleInput Multiple-Output (MIMO) $W\left(T_{s}\right)$ as:

$$
\begin{array}{lll}
\forall i \in\left\{1, \ldots, N_{U}\right\}, & \forall \omega \in \Omega_{U}^{i}, & \bar{\sigma}\left(W\left(T_{s}(j \omega)\right)\right)<U_{i} \\
\forall l \in\left\{1, \ldots, N_{L}\right\}, & \forall \omega \in \Omega_{L}^{l}, & \underline{\sigma}\left(W\left(T_{s}(j \omega)\right)\right)>L_{l}
\end{array}
$$

The following approach holds for general MIMO $W\left(T_{s}\right)$. However, since usually SISO $W\left(T_{s}\right)$ are of practical interest, design problems are presented in the SISO case.

\section{Discrete-Mask Design Problem}

Problem 2 (Discrete-Mask Formulation): Given $N$ positive real numbers $W_{i}$ and frequencies $\omega_{i}$,

Minimize $\lambda$ such that there exists a stable identical dissipative systems interconnection $W\left(T_{s}\right)$, such that:

$$
\sum_{i=1}^{N}\left(\left|W\left(T_{s}\left(j \omega_{i}\right)\right)\right|^{2}-W_{i}^{2}\right)^{2} \leq \lambda^{2}
$$

and compute $W\left(T_{s}\right)$.

\section{PROposed APPROACH}

When $T_{s}(s)=1 / s$, Problem 1 and 2 are solved in two steps [11], [12]: $(i)$ the squared magnitude $M(\omega)$ of the frequency response $W(j \omega)$ is computed, $(i i) W(s)$ is computed such that $|W(j \omega)|^{2}=M(\omega)$. The motivation is that while condition (5), for instance, is not convex in the variable $W$, the change of variable from $W$ to $M$ allows to recast the computation of a solution as a convex optimisation problem (step $(i)$ ). The purpose of step $(i i)$, known as spectral factorisation, is then to obtain $W$ from $M$.

The resulting problem is a convex optimisation problem but infinite dimensional. First, as the decision variable $M$ is a rational function of $\omega$, it belongs to an infinite dimensional space. To overcome this, $M$ is factorised as $M(\omega)=\frac{\mathcal{B}(j \omega)^{*} \mathcal{X}_{N} \mathcal{B}(j \omega)}{\mathcal{B}(j \omega)^{*} \mathcal{X}_{D} \mathcal{B}(j \omega)}$, with $\mathcal{X}_{N}=\mathcal{X}_{N}^{T}$ and $\mathcal{X}_{D}=\mathcal{X}_{D}^{T}$ two real symmetric matrices, and $\mathcal{B}(s)$ a chosen basis of polynomials in $s$. The decision variables $\mathcal{X}_{N}$ and $\mathcal{X}_{D}$ are then in a finite dimensional space. Second, there is an 
infinite number of constraints as they depend on $\omega$. Applying the so-called KYP Lemma and its extensions [13] to these constraints, a finite convex optimisation is finally obtained.

In the rest of this section, we investigate how this approach can be generalised to Problem 1 and 2. In subsection IV-A, an alternative version of the KYP Lemma is applied to extend step $(i)$ to identical dissipative systems interconnection. In subsection IV-B, a dissipative version of the spectral factorisation theorem extends step $(i i)$. In subsection IV$\mathrm{C}$, it appears that it is not possible to obtain a solution by generalising each step independently for lossy dissipative $T_{s}$. We then reveal that a general solution of Problem 1 and 2 can be convexly computed by coupling both steps.

\section{A. Step $(i)$ : Magnitude Design}

This subsection presents the extension of step $(i)$ to identical dissipative systems interconnection for continuousand discrete-spectral masks. Both results are based on the following lemma.

Lemma 1: Let $\mathcal{I}$ be either $\mathbb{R}$ or a sub-interval of $\mathbb{R}$. Assume $T_{s}$ is $\{x, y, z\}$-dissipative on $\mathcal{I}$. Let $\mathcal{X}=\mathcal{X}^{T} \in \mathbb{R}^{n_{z} \times n_{z}}$ be a real symmetric matrix. Let $\mathcal{B}\left(T_{s}\right)$ be described by the LFT: $\mathcal{B}\left(T_{s}\right):=\left(T_{s} \cdot I_{n}\right) \star\left[\begin{array}{c|c}A & B \\ \hline C & D\end{array}\right]$, where $A \in \mathbb{R}^{n \times n}, B \in \mathbb{R}^{n \times n_{w}}, C \in \mathbb{R}^{n_{z} \times n}, D_{B} \in \mathbb{R}^{n_{z} \times n_{w}}$. Then $(i)$ implies $(i i)$ :

(i) There exists $P=P^{T}>0 \in \mathbb{R}^{n \times n}$ such that

$$
\mathcal{L} \mathcal{M}\left(\left[\begin{array}{ll}
A & B \\
C & D
\end{array}\right],\left[\begin{array}{ll}
-z P & -y P \\
-y P & -x P
\end{array}\right], \mathcal{X}\right)>0
$$

(ii) The condition (7) is satisfied:

$$
\forall \omega \in \mathcal{I}, \quad \mathcal{B}\left(T_{s}(j \omega)\right)^{*} \mathcal{X} \mathcal{B}\left(T_{s}(j \omega)\right)>0
$$

Proof: By definition of positive definite matrices, inequality (6) is equivalent to: $\forall w \in \mathbb{C}^{n_{w}}, \forall p \in \mathbb{C}^{n}$,

$$
\left[\begin{array}{c}
p \\
w
\end{array}\right]^{*} \mathcal{L} \mathcal{M}\left(\left[\begin{array}{ll}
A & B \\
C & D
\end{array}\right],\left[\begin{array}{ll}
-z P & -y P \\
-y P & -x P
\end{array}\right], \mathcal{X}\right)\left[\begin{array}{c}
p \\
w
\end{array}\right]>0
$$

Defining the signal $q \in \mathbb{C}^{n}$ as in the LFR (1), one has:

$$
\underbrace{\left[\begin{array}{l}
q \\
p
\end{array}\right]^{*}\left[\begin{array}{ll}
-z P & -y P \\
-y P & -x P
\end{array}\right]\left[\begin{array}{l}
q \\
p
\end{array}\right]}_{\leq 0}+\left[\begin{array}{l}
p \\
w
\end{array}\right]^{*}\left[\begin{array}{l}
C^{T} \\
D^{T}
\end{array}\right] \mathcal{X}\left[\begin{array}{ll}
C & D
\end{array}\right]\left[\begin{array}{l}
p \\
w
\end{array}\right]>0
$$

as, by setting $p=\left(T_{s} \cdot I_{n}\right) q$ and by assumption: $\forall \omega \in \mathcal{I}$,

$$
\begin{aligned}
& {\left[\begin{array}{c}
q \\
p
\end{array}\right]^{*}\left[\begin{array}{cc}
-z P & -y P \\
-y P & -x P
\end{array}\right]\left[\begin{array}{l}
q \\
p
\end{array}\right]=-\left[\begin{array}{l}
p \\
q
\end{array}\right]^{*}\left[\begin{array}{ll}
x P & y P \\
y P & z P
\end{array}\right]\left[\begin{array}{l}
p \\
q
\end{array}\right] } \\
= & -q^{*}\left[\begin{array}{c}
T_{s}(j \omega) \cdot I \\
I
\end{array}\right]^{*}\left[\begin{array}{ll}
x P & y P \\
y P & z P
\end{array}\right]\left[\begin{array}{c}
T_{s}(j \omega) \cdot I \\
I
\end{array}\right] q \leq 0
\end{aligned}
$$

Finally, as $q=\left(I_{n}-A T_{s}(j \omega)\right)^{-1} B w$, little calculations from (8) gives: $\forall w \in \mathbb{C}^{n_{w}}, \forall \omega \in \mathcal{I}$, $w^{*}\left(\mathcal{B}\left(T_{s}(j \omega)\right)^{*} \mathcal{X} \mathcal{B}\left(T_{s}(j \omega)\right)\right) w>0$. which implies (7).

Remark 3: (6) is an LMI in the matrices $P$ and $\mathcal{X}$.

Remark 4: If $T_{s}(s)=1 / s$ and $\mathcal{I}=\mathbb{R}$, Lemma 1 matches with the KYP lemma. When $\mathcal{I}$ is a sub-interval of $\mathbb{R}$, it matches extended versions of the KYP lemma [13].
In the rest of this subsection, continuous- and discretemask magnitude design problems are formulated as feasibility/linear minimisation problems under LMI constraints.

\section{1) Continuous-Mask Magnitude Design:}

Theorem 1: Assume that $T_{s}$ is $\left\{x_{d}, y_{d}, z_{d}\right\}$-dissipative on $\mathbb{R},\left\{x_{i}, y_{i}, z_{i}\right\}$-dissipative on $\Omega_{U}^{i}$ and $\left\{x_{l}, y_{l}, z_{l}\right\}$-dissipative on $\Omega_{L}^{l}$. Let $\mathcal{X}_{N}=\mathcal{X}_{N}^{T} \in \mathbb{R}^{n_{z} \times n_{z}}$ and $\mathcal{X}_{D}=\mathcal{X}_{D}^{T} \in \mathbb{R}^{n_{z} \times n_{z}}$ be real symmetric matrices. Let $\mathcal{B}_{B}\left(T_{s}\right)$ be as:

$$
\mathcal{B}_{B}\left(T_{s}\right):=\left(T_{s} \cdot I_{n}\right) \star\left[\begin{array}{c|c}
A_{B} & B_{B} \\
\hline C_{B} & D_{B}
\end{array}\right]
$$

where $A_{B} \in \mathbb{R}^{n \times n}, B_{B} \in \mathbb{R}^{n \times 1}, C_{B} \in \mathbb{R}^{n_{z} \times n}, D_{B} \in \mathbb{R}^{n_{z} \times 1}$. If there exist real, positive matrices $P_{N}=P_{N}^{T} \in \mathbb{R}^{n \times n}>0, \quad P_{D}=P_{D}^{T} \in \mathbb{R}^{n \times n}>0$, $P_{i}=P_{i}^{T} \in \mathbb{R}^{n \times n}>0, P_{l}=P_{l}^{T} \in \mathbb{R}^{n \times n}>0$ such that:

$$
\begin{aligned}
& \mathcal{L} \mathcal{M}\left(\left[\begin{array}{ll}
A_{B} & B_{B} \\
C_{B} & D_{B}
\end{array}\right],\left[\begin{array}{ll}
-z_{d} P_{N} & -y_{d} P_{N} \\
-y_{d} P_{N} & -x_{d} P_{N}
\end{array}\right], \mathcal{X}_{N}\right)>0 \\
& \mathcal{L} \mathcal{M}\left(\left[\begin{array}{ll}
A_{B} & B_{B} \\
C_{B} & D_{B}
\end{array}\right],\left[\begin{array}{ll}
-z_{d} P_{D} & -y_{d} P_{D} \\
-y_{d} P_{D} & -x_{d} P_{D}
\end{array}\right], \mathcal{X}_{D}\right)>0
\end{aligned}
$$

$\forall i \in\left\{1, \ldots, N_{U}\right\}$,

$\mathcal{L} \mathcal{M}\left(\left[\begin{array}{ll}A_{B} & B_{B} \\ C_{B} & D_{B}\end{array}\right],\left[\begin{array}{ll}-z_{i} P_{i} & -y_{i} P_{i} \\ -y_{i} P_{i} & -x_{i} P_{i}\end{array}\right], U_{i}^{2} \mathcal{X}_{D}-\mathcal{X}_{N}\right)>0$

$\forall l \in\left\{1, \ldots, N_{L}\right\}$,

$\mathcal{L} \mathcal{M}\left(\left[\begin{array}{ll}A_{B} & B_{B} \\ C_{B} & D_{B}\end{array}\right],\left[\begin{array}{ll}-z_{l} P_{l} & -y_{l} P_{l} \\ -y_{l} P_{l} & -x_{l} P_{l}\end{array}\right], \mathcal{X}_{N}-L_{l}^{2} \mathcal{X}_{D}\right)>0$

Then

$$
\begin{aligned}
& \forall i \in\left\{1, \ldots, N_{U}\right\}, \forall \omega \in \Omega_{U}^{i}, \frac{M_{N}\left(T_{s}(j \omega)\right)}{M_{D}\left(T_{s}(j \omega)\right)}<U_{i}^{2} \\
& \forall l \in\left\{1, \ldots, N_{L}\right\}, \forall \omega \in \Omega_{L}^{l}, \frac{M_{N}\left(T_{s}(j \omega)\right)}{M_{D}\left(T_{s}(j \omega)\right)}>L_{l}^{2}
\end{aligned}
$$

hold, where $\forall \omega \in \mathbb{R}$,

$$
\begin{aligned}
& M_{N}\left(T_{s}(j \omega)\right)=\mathcal{B}_{B}\left(T_{s}(j \omega)\right)^{*} \mathcal{X}_{N} \mathcal{B}_{B}\left(T_{s}(j \omega)\right) \\
& M_{D}\left(T_{s}(j \omega)\right)=\mathcal{B}_{B}\left(T_{s}(j \omega)\right)^{*} \mathcal{X}_{D} \mathcal{B}_{B}\left(T_{s}(j \omega)\right)
\end{aligned}
$$

Proof: Conditions (10) - (13) imply by Lemma 1 that:

$$
\begin{aligned}
\forall \omega \in \mathbb{R}, & \mathcal{B}_{B}\left(T_{s}(j \omega)\right)^{*} \mathcal{X}_{N} \mathcal{B}_{B}\left(T_{s}(j \omega)\right)>0 \\
\forall \omega \in \mathbb{R}, & \mathcal{B}_{B}\left(T_{s}(j \omega)\right)^{*} \mathcal{X}_{D} \mathcal{B}_{B}\left(T_{s}(j \omega)\right)>0 \\
\forall \omega \in \Omega_{U}^{i}, & \mathcal{B}_{B}\left(T_{s}(j \omega)\right)^{*}\left(U_{i}^{2} \mathcal{X}_{D}-\mathcal{X}_{N}\right) \mathcal{B}_{B}\left(T_{s}(j \omega)\right)>0 \\
\forall \omega \in \Omega_{L}^{l}, & \mathcal{B}_{B}\left(T_{s}(j \omega)\right)^{*}\left(\mathcal{X}_{N}-L_{l}^{2} \mathcal{X}_{D}\right) \mathcal{B}_{B}\left(T_{s}(j \omega)\right)>0
\end{aligned}
$$

Mere calculation gives then (14) and (15).

2) Discrete-Mask Magnitude Design: In the discretemask formulation, it is aimed to find the squared magnitudes $M_{N}\left(T_{s}\right)$ and $M_{N}\left(T_{s}\right)$ such that: $\lambda$ is minimised and

$$
\sum_{i=1}^{N}\left(\frac{M_{N}\left(T_{s}\left(j \omega_{i}\right)\right)}{M_{D}\left(T_{s}\left(j \omega_{i}\right)\right)}-W_{i}^{2}\right)^{2} \leq \lambda^{2}
$$

is satisfied. As (18) is generally not convex, an alternative problem is to minimise $\lambda$ such that

$$
\sum_{i=1}^{N} Q_{i}^{2}\left(\frac{M_{N}\left(T_{s}\left(j \omega_{i}\right)\right)}{M_{D}\left(T_{s}\left(j \omega_{i}\right)\right)}-W_{i}^{2}\right)^{2} \leq \lambda^{2}
$$


is verified. Then, by choosing $Q_{i}=M_{D}\left(T_{s}\left(j \omega_{i}\right)\right)$, this alternative (suboptimal) problem becomes convex in the decision variables $M_{N}$ and $M_{D}$. This problem then becomes: minimise $\lambda$ such that (19) hold:

$$
\sum_{i=1}^{N}\left(M_{N}\left(T_{s}\left(j \omega_{i}\right)\right)-W_{i}^{2} M_{D}\left(T_{s}\left(j \omega_{i}\right)\right)\right)^{2} \leq \lambda^{2}
$$

Theorem 2: Assume that $T_{s}$ is $\left\{x_{d}, y_{d}, z_{d}\right\}$-dissipative on $\mathbb{R}$. Let $\mathcal{X}_{N}=\mathcal{X}_{N}^{T} \in \mathbb{R}^{n_{z} \times n_{z}}$ and $\mathcal{X}_{D}=\mathcal{X}_{D}^{T} \in \mathbb{R}^{n_{z} \times n_{z}}$ be real symmetric matrices. Let $\mathcal{B}_{B}$ be as in (9). Let $\lambda>0 \in \mathbb{R}$ be a real positive scalar.

If there exist $P_{N}=P_{N}^{T} \in \mathbb{R}^{n \times n}>0, P_{D}=P_{D}^{T} \in$ $\mathbb{R}^{n \times n}>0$, such that: (10), (11) and (20) are satisfied:

$$
\left[\begin{array}{ccccc}
\lambda & \Gamma_{1} & \Gamma_{2} & \ldots & \Gamma_{N} \\
\Gamma_{1} & \lambda & 0 & \ldots & 0 \\
\Gamma_{2} & 0 & \ddots & \ddots & \vdots \\
\vdots & \vdots & \ddots & \lambda & 0 \\
\Gamma_{N} & 0 & \ldots & 0 & \lambda
\end{array}\right] \geq 0
$$

with $\Gamma_{i}:=\mathcal{B}_{B}\left(T_{s}\left(j \omega_{i}\right)\right)^{*}\left(\mathcal{X}_{N}-W_{i}^{2} \mathcal{X}_{D}\right) \mathcal{B}_{B}\left(T_{s}\left(j \omega_{i}\right)\right)$ Then condition (19) holds, with $M_{N}\left(T_{s}\right)$ and $M_{D}\left(T_{s}\right)$ defined respectively by (16) and (17).

Proof: By applying successively the Schur complement Lemma [14], (19) becomes equivalent to (20).

\section{B. Step (ii): Dissipative Spectral Factorisation}

The following theorem extends the spectral factorisation theorem from state-space systems to identical dissipative systems interconnections.

Theorem 3 (Dissipative Spectral Factorisation): Assume that $T_{s}$ is $\left\{x_{d}, y_{d}, z_{d}\right\}$-dissipative on $\mathbb{R}$. Let $\mathcal{B}_{B}\left(T_{s}\right)$ be as in (9). Let $\widehat{A}_{B}, \widehat{B}_{B}, \widehat{C}_{B}$ and $\widehat{D}_{B}$ be defined as in Lemma 2. Let $\mathcal{X}=\mathcal{X}^{T} \in \mathbb{R}^{n_{z} \times n_{z}}$ and $P=P^{T} \in \mathbb{R}^{n \times n}>0$ be such that

$$
\mathcal{L M}\left(\left[\begin{array}{ll}
A_{B} & B_{B} \\
C_{B} & D_{B}
\end{array}\right],\left[\begin{array}{ll}
-z_{d} P & -y_{d} P \\
-y_{d} P & -x_{d} P
\end{array}\right], \mathcal{X}\right)>0
$$

and $\widehat{D}_{B}^{T} \mathcal{X} \widehat{D}_{B}>0$ are verified. Let $P^{R}=\left(P^{R}\right)^{T} \in \mathbb{R}^{n \times n}$.

If $P^{R}$ is solution to the Algebraic Riccati Equation (ARE):

$$
\mathcal{R}\left(\left[\begin{array}{ll}
\widehat{A}_{B} & \widehat{B}_{B} \\
\widehat{C}_{B} & \widehat{D}_{B}
\end{array}\right], P^{R}, \mathcal{X}\right)=0
$$

Then there exist $C_{W} \in \mathbb{R}^{n_{w} \times n}$ and $D_{W} \in \mathbb{R}^{n_{w} \times n_{w}}$ such that $W\left(T_{s}\right)$, defined by (22), is stable and satisfies: $\forall \omega \in \mathbb{R}$,

$$
\begin{aligned}
& W\left(T_{s}(j \omega)\right)^{*} W\left(T_{s}(j \omega)\right) \\
& \begin{array}{l}
=\mathcal{B}_{B}\left(T_{s}(j \omega)\right)^{*} \mathcal{X} \mathcal{B}_{B}\left(T_{s}(j \omega)\right)-\mathcal{F}_{B}\left(T_{s}(j \omega)\right)^{*} E_{d}^{R} \\
\text { where } E_{d}^{R} \text { is defined by (23) and } \mathcal{F}_{B}\left(T_{s}\right) \text { by }(24) \\
\qquad W\left(T_{s}\right):=\left(T_{s} \cdot I_{n}\right) \star\left[\begin{array}{c|c}
A_{B} & B_{B} \\
\hline C_{W} & D_{W}
\end{array}\right]
\end{array} \\
& E_{d}^{R}:=\left[\begin{array}{ll}
x_{d} P^{R} & y_{d} P^{R} \\
y_{d} P^{R} & z_{d} P^{R}
\end{array}\right] \\
& \mathcal{F}_{B}\left(T_{s}\right)=\left(T_{s} \cdot I_{n}\right) \star\left[\begin{array}{c|c}
A_{B} & B_{B} \\
\hline I_{n} & 0 \\
A_{B} & B_{B}
\end{array}\right]
\end{aligned}
$$

The proof is based on Lemma 2.

Lemma 2: The following matrices are congruent:

$$
\begin{aligned}
& Q(P):=\mathcal{L} \mathcal{M}\left(\left[\begin{array}{ll}
A_{B} & B_{B} \\
C_{B} & D_{B}
\end{array}\right],\left[\begin{array}{cc}
-z_{d} P & -y_{d} P \\
-y_{d} P & -x_{d} P
\end{array}\right], \mathcal{X}\right) \\
& \widehat{Q}(P):=\mathcal{L} \mathcal{M}\left(\left[\begin{array}{ll}
\widehat{A}_{B} & \widehat{B}_{B} \\
\widehat{C}_{B} & \widehat{D}_{B}
\end{array}\right],\left[\begin{array}{cc}
0 & -P \\
-P & 0
\end{array}\right], \mathcal{X}\right)
\end{aligned}
$$

with, by defining $r:=\sqrt{y_{d}^{2}-x_{d} z_{d}}$ :

$$
\left[\begin{array}{c|c}
\widehat{A}_{B} & \widehat{B}_{B} \\
\hline \widehat{C}_{B} & \widehat{D}_{B}
\end{array}\right]=\left[\begin{array}{c|c}
\frac{\frac{y_{d}+r}{x_{d}} I}{2\left(y_{d}+r\right)} & \frac{-\sqrt{2\left(y_{d}+r\right)}}{x_{d}} I \\
\hline \frac{r \sqrt{x_{d}}}{x_{d}} I & \frac{-\left(y_{d}+r\right)}{x_{d}} I
\end{array}\right] \star\left[\begin{array}{c|c}
A & B \\
\hline \frac{-C}{r} & \frac{-D}{r}
\end{array}\right]
$$

Let now prove Theorem 3 .

Proof: Let $k \in \mathbb{N}$ such that $k \geq n_{w}$. Let $C_{W} \in \mathbb{R}^{k \times n}$ and $D_{W} \in \mathbb{R}^{k \times n_{w}}$ be such that:

$$
Q\left(P^{R}\right)=\left[\begin{array}{ll}
C_{W} & D_{W}
\end{array}\right]^{T}\left[\begin{array}{ll}
C_{W} & D_{W}
\end{array}\right]
$$

Let prove that one can set $k=n_{w}$. By Lemma 2, $Q\left(P^{R}\right)$ and $\widehat{Q}\left(P^{R}\right)$ are congruent. Then, they have same rank [14]: $\operatorname{rank}\left(Q\left(P^{R}\right)\right)=\operatorname{rank}\left(\widehat{Q}\left(P^{R}\right)\right)$.

By Schur complement Lemma, $\widehat{Q}\left(P^{R}\right)$ is congruent with:

$$
\widehat{Q}^{S}\left(P^{R}\right):=\left[\begin{array}{cc}
\mathcal{R}\left(\left[\begin{array}{cc}
\widehat{A}_{B} & \widehat{B}_{B} \\
\widehat{C}_{B} & \widehat{D}_{B}
\end{array}\right], P^{R}, \mathcal{X}\right) & 0 \\
0 & \widehat{D}_{B}^{T} \mathcal{X} \widehat{D}_{B}
\end{array}\right]
$$

If $P^{R}$ is solution of (21), by congruency of matrices: $\operatorname{rank}\left(Q\left(P^{R}\right)\right)=\operatorname{rank}\left(\widehat{D}_{B}^{T} \mathcal{X} \widehat{D}_{B}\right)=n_{w}$. Thus by (25):

$$
\operatorname{rank}\left(\left[\begin{array}{c}
C_{W}^{T} \\
D_{W}^{T}
\end{array}\right]\left[\begin{array}{ll}
C_{W} & D_{W}
\end{array}\right]\right)=\operatorname{rank}\left[\begin{array}{ll}
C_{W} & D_{W}
\end{array}\right]=n_{w}
$$

Therefore, one can find $C_{W}, D_{W}$ such that $k=n_{w}$.

Finally, $\forall w \in \mathbb{C}$, setting $p$ as in the LFR (1):

$$
\left[\begin{array}{c}
p \\
w
\end{array}\right]^{*} Q\left(P^{R}\right)\left[\begin{array}{c}
p \\
w
\end{array}\right]=\left[\begin{array}{c}
p \\
w
\end{array}\right]^{*}\left[\begin{array}{ll}
C_{W} & D_{W}
\end{array}\right]^{T}\left[\begin{array}{ll}
C_{W} & D_{W}
\end{array}\right]\left[\begin{array}{c}
p \\
w
\end{array}\right]
$$

a little bit of calculation ends up with (22)-(24).

1) Discussion on the factorisation: When $T_{s}$ is lossless dissipative, the factor $\mathcal{F}_{B}\left(T_{s}(j \omega)\right)^{*} E_{d} \mathcal{F}_{B}\left(T_{s}(j \omega)\right)$ is equal to zero as:

$\forall \omega \in \mathbb{R},\left[\begin{array}{c}T_{s}(j \omega) \cdot I_{n} \\ I_{n}\end{array}\right]^{*}\left[\begin{array}{ll}x_{d} P^{R} & y_{d} P^{R} \\ y_{d} P^{R} & z_{d} P^{R}\end{array}\right]\left[\begin{array}{c}T_{s}(j \omega) \cdot I_{n} \\ I_{n}\end{array}\right]=0$ The factorisation is said to be lossless, meaning that $W(T)$ is computed such that $|W(T(j \omega))|^{2}=M(T(j \omega))$.

When $T_{s}$ is lossy dissipative, the lossy factor $\mathcal{F}_{B}\left(T_{s}(j \omega)\right)^{*} E_{d}^{R} \mathcal{F}_{B}\left(T_{s}(j \omega)\right)$ is non-zero. There is then a mismatch between $\left|W\left(T_{s}(j \omega)\right)\right|^{2}$ and $M\left(T_{s}(j \omega)\right)$. The conventional two-steps approach can not be applied. In Subsection IV-C, we develop a new approach able to cope with lossy dissipative $T_{s}$. 
2) Discussion on implementation: The spectral factorisation requires to solve the ARE (21). The extreme positive semi-definite solutions $P_{\min }^{R}$ and $P_{\max }^{R}$ of the ARE are the extreme positive semi-definite solutions of the set of solutions $P$ of the LMI $\widehat{Q}(P) \geq 0$ [15], [16]. In the sequel, only $P_{\text {min }}^{R}$ will be considered, as it corresponds to minimumphase filter in the usual state-space approach. As $\widehat{Q}(P)$ and $Q(P)$ are congruent, $P_{\min }^{R}$ is obtained by minimising the trace of the decision variable $P$ over $Q(P)>0$.

\section{Coupled Design-Factorisation Approach}

The idea behind the coupled design-factorisation approach is to include the lossy factor into the magnitude design problem. The frequency constraints are then set on $\left|W\left(T_{s}(j \omega)\right)\right|^{2}=B\left(T_{s}\right) * \mathcal{X} B\left(T_{s}\right)-\mathcal{F}_{B}\left(T_{s}\right)^{*} E_{d}^{R} \mathcal{F}_{B}\left(T_{s}\right)$.

Problem 1 and Problem 2 are separately considered.

1) Continuous-Mask Design Solution: A solution of Problem 1 is given in the following theorem.

Theorem 4: Let $\mathcal{B}_{B}\left(T_{s}\right)$ be as in (9). Define $\widehat{A}_{B}, \widehat{B}_{B}, \widehat{C}_{B}$ and $\widehat{D}_{B}$ as in Lemma 2 .

Then the solution of the optimisation problem $(i)$ gives a solution to problem $(i i)$.

(i)

$$
\begin{aligned}
& \min _{\boldsymbol{X}_{N}=\mathcal{X}_{N}^{T}, \mathcal{X}_{D}=\mathcal{X}_{D}^{T}}\left\{\operatorname{trace}\left(\boldsymbol{P}_{\boldsymbol{N}}\right)+\operatorname{trace}\left(\boldsymbol{P}_{\boldsymbol{D}}\right)\right\} \\
& \boldsymbol{P}_{\boldsymbol{N}}=\boldsymbol{P}_{N}^{T}>0, \boldsymbol{P}_{\boldsymbol{D}}=\boldsymbol{P}_{D}^{T}>0 \\
& \boldsymbol{P}_{\boldsymbol{i}}=\boldsymbol{P}_{i}^{T}>0, \boldsymbol{P}_{\boldsymbol{l}}=\boldsymbol{P}_{l}^{T}>0 \\
& \text { such that (10), (11), (26), (27), (28) hold: }
\end{aligned}
$$

$$
\begin{aligned}
& \widehat{D}_{B}^{T} \mathcal{X}_{\boldsymbol{N}} \widehat{D}_{B}>0 \quad \widehat{D}_{B}^{T} \mathcal{X}_{\boldsymbol{D}} \widehat{D}_{B}>0 \\
& \mathcal{L} \mathcal{M}\left(\left[\begin{array}{ll}
A_{B} & B_{B} \\
\bar{C}_{B} & \bar{D}_{B}
\end{array}\right],\left[\begin{array}{ll}
-z_{i} \boldsymbol{P}_{\boldsymbol{i}} & -y_{i} \boldsymbol{P}_{\boldsymbol{i}} \\
-y_{i} \boldsymbol{P}_{\boldsymbol{i}} & -x_{i} \boldsymbol{P}_{\boldsymbol{i}}
\end{array}\right],\right. \\
& \left.\left[\begin{array}{cc}
U_{i}^{2} \mathcal{X}_{\boldsymbol{D}}-\mathcal{X}_{\boldsymbol{N}} & 0 \\
0 & \boldsymbol{E}_{\boldsymbol{d}_{\boldsymbol{N}}}-U_{i}^{2} \boldsymbol{E}_{\boldsymbol{d}_{\boldsymbol{D}}}
\end{array}\right]\right)>0 \\
& \mathcal{L} \mathcal{M}\left(\left[\begin{array}{ll}
A_{B} & B_{B} \\
\bar{C}_{B} & \bar{D}_{B}
\end{array}\right],\left[\begin{array}{ll}
-z_{l} \boldsymbol{P}_{\boldsymbol{l}} & -y_{l} \boldsymbol{P}_{\boldsymbol{l}} \\
-y_{l} \boldsymbol{P}_{\boldsymbol{l}} & -x_{l} \boldsymbol{P}_{\boldsymbol{l}}
\end{array}\right],\right. \\
& \left.\left[\begin{array}{cc}
\mathcal{X}_{\boldsymbol{N}}-L_{l}^{2} \mathcal{X}_{\boldsymbol{D}} & 0 \\
0 & L_{l}^{2} \boldsymbol{E}_{\boldsymbol{d}_{\boldsymbol{D}}}-\boldsymbol{E}_{\boldsymbol{d}_{\boldsymbol{N}}}
\end{array}\right]\right)>0
\end{aligned}
$$

with $\bar{C}_{B}=\left[\begin{array}{lll}C_{B}^{T} & I_{n} & A_{B}^{T}\end{array}\right]^{T}, \bar{D}_{B}^{T}=\left[\begin{array}{lll}D_{B}^{T} & 0 & B_{B}^{T}\end{array}\right]^{T}$, $\boldsymbol{E}_{\boldsymbol{d}_{\boldsymbol{N}}}=\left[\begin{array}{ll}x_{d} \boldsymbol{P}_{\boldsymbol{N}} & y_{d} \boldsymbol{P}_{\boldsymbol{N}} \\ y_{d} \boldsymbol{P}_{\boldsymbol{N}} & z_{d} \boldsymbol{P}_{\boldsymbol{N}}\end{array}\right]$ and $\boldsymbol{E}_{\boldsymbol{d}_{\boldsymbol{D}}}=\left[\begin{array}{ll}x_{d} \boldsymbol{P}_{\boldsymbol{D}} & y_{d} \boldsymbol{P}_{\boldsymbol{D}} \\ y_{d} \boldsymbol{P}_{\boldsymbol{D}} & z_{d} \boldsymbol{P}_{\boldsymbol{D}}\end{array}\right]$.

(ii) Find real matrices $C_{N}, C_{D} \in \mathbb{R}^{1 \times n}$ and $D_{N}, D_{D} \in \mathbb{R}^{1 \times 1}$ such that the identical dissipative systems interconnection $W\left(T_{s}\right)=W_{N}\left(T_{s}\right)\left(W_{D}\left(T_{s}\right)\right)^{-1}$ is stable, with

$$
\begin{aligned}
& W_{N}\left(T_{s}\right)=\left(T_{s} \cdot I_{n}\right) \star\left[\begin{array}{c|c}
A_{B} & B_{B} \\
\hline C_{N} & D_{N}
\end{array}\right] \\
& W_{D}\left(T_{s}\right)=\left(T_{s} \cdot I_{n}\right) \star\left[\begin{array}{l|l}
A_{B} & B_{B} \\
\hline C_{D} & D_{D}
\end{array}\right]
\end{aligned}
$$

and satisfies (4) and (5).

Proof: As the trace of $P_{N}$ and $P_{D}$ are minimised under (10), (11), by Theorem 3 and Subsection IV-B.2:

$$
\begin{aligned}
& \exists C_{N}, C_{D} \in \mathbb{R}^{1 \times n}, \exists D_{N}, D_{D} \in \mathbb{R}^{1 \times 1}, \forall \omega \in \mathbb{R}, \\
& \mathcal{B}_{B}\left(T_{s}(j \omega)\right)^{*} \mathcal{X}_{N} \mathcal{B}_{B}\left(T_{s}(j \omega)\right)-\mathcal{F}_{B}\left(T_{s}(j \omega)\right)^{*} E_{d_{N}} \mathcal{F}_{B}\left(T_{s}(j \omega)\right. \\
& =W_{N}\left(T_{s}(j \omega)\right)^{*} W_{N}\left(T_{s}(j \omega)\right) \\
& \mathcal{B}_{B}\left(T_{s}(j \omega)\right)^{*} \mathcal{X}_{D} \mathcal{B}_{B}\left(T_{s}(j \omega)\right)-\mathcal{F}_{B}\left(T_{s}(j \omega)\right)^{*} E_{d_{D}} \mathcal{F}_{B}\left(T_{s}(j \omega)\right. \\
& =W_{D}\left(T_{s}(j \omega)\right)^{*} W_{D}\left(T_{s}(j \omega)\right)
\end{aligned}
$$

with $\mathcal{F}_{B}\left(T_{s}\right)$ defined by (24). Moreover, (27) and (28) imply by Lemma 1: $\forall \omega \in \Omega_{U}^{i}$,

$$
\begin{aligned}
\mathcal{F}_{B}\left(T_{s}(j \omega)\right)^{*} & \left(E_{d_{N}}-U_{i}^{2} E_{d_{D}}\right) \mathcal{F}_{B}\left(T_{s}(j \omega)\right) \\
& +\mathcal{B}_{B}\left(T_{s}(j \omega)\right)^{*}\left(U_{i}^{2} \mathcal{X}_{D}-\mathcal{X}_{N}\right) \mathcal{B}_{B}\left(T_{s}(j \omega)\right)>0
\end{aligned}
$$

and $\forall \omega \in \Omega_{L}^{l}$,

$$
\begin{aligned}
\mathcal{F}_{B}\left(T_{s}(j \omega)\right)^{*} & \left(L_{l}^{2} E_{d_{D}}-E_{d_{N}}\right) \mathcal{F}_{B}\left(T_{s}(j \omega)\right) \\
& +\mathcal{B}_{B}\left(T_{s}(j \omega)\right)^{*}\left(\mathcal{X}_{N}-L_{l}^{2} \mathcal{X}_{D}\right) \mathcal{B}_{B}\left(T_{s}(j \omega)\right)>0
\end{aligned}
$$

Then little bit of calculation implies that (4) and (5) hold.

2) Discrete-Mask Design Solution: A solution of Problem 2 is given in the following theorem.

Theorem 5: Let $\mathcal{B}_{B}\left(T_{s}\right)$ be as in (9). Define $\widehat{A}_{B}, \widehat{B}_{B}, \widehat{C}_{B}$ and $\widehat{D}_{B}$ as in Lemma 2 .

Then the solution of the optimisation problem $(i)$ gives a solution to problem $(i i)$.

(i)

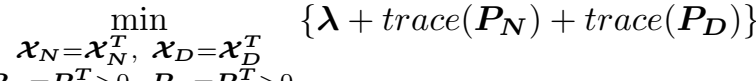

$$
\begin{aligned}
& \boldsymbol{P}_{\boldsymbol{N}}=\boldsymbol{P}_{N}^{T}>0, \boldsymbol{P}_{D}=\boldsymbol{P}_{D}^{T}>0
\end{aligned}
$$

$$
\left[\begin{array}{ccccc}
\lambda & \overline{\boldsymbol{\Gamma}}_{1} & \overline{\boldsymbol{\Gamma}}_{\mathbf{2}} & \ldots & \overline{\boldsymbol{\Gamma}}_{\boldsymbol{N}} \\
\overline{\boldsymbol{\Gamma}}_{\mathbf{1}} & \boldsymbol{\lambda} & 0 & \ldots & 0 \\
\overline{\boldsymbol{\Gamma}}_{\mathbf{2}} & 0 & \ddots & \ddots & \vdots \\
\vdots & \vdots & \ddots & \lambda & 0 \\
\overline{\boldsymbol{\Gamma}}_{\boldsymbol{N}} & 0 & \ldots & 0 & \lambda
\end{array}\right] \geq 0
$$

with $\overline{\boldsymbol{\Gamma}}_{\boldsymbol{i}}=\boldsymbol{\Gamma}_{\boldsymbol{i}}-\mathcal{F}_{B}\left(T\left(j \omega_{i}\right)\right)^{*}\left(W_{i}^{2} \boldsymbol{E}_{\boldsymbol{d}_{\boldsymbol{D}}}-\boldsymbol{E}_{\boldsymbol{d}_{\boldsymbol{N}}}\right) \mathcal{F}_{B}\left(T\left(j \omega_{i}\right)\right)$

(ii) Find real matrices $C_{N}, C_{D} \in \mathbb{R}^{1 \times n}$ and $D_{N}, D_{D} \in \mathbb{R}^{1 \times 1}$ such that the identical dissipative systems interconnection $W\left(T_{s}\right)=W_{N}\left(T_{s}\right)\left(W_{D}\left(T_{s}\right)\right)^{-1}$ is stable, with $W_{N}\left(T_{s}\right)$ and $W_{D}\left(T_{s}\right)$ defined by (29), (30), and satisfies

$$
\begin{aligned}
& \sum_{\substack{i=1 \\
\text { Proof: Similar proof as in the continuous case. }}}^{N}\left(\left|W_{N}\left(T_{s}\left(j \omega_{i}\right)\right)\right|^{2}-W_{i}^{2}\left|W_{D}\left(T_{s}\left(j \omega_{i}\right)\right)\right|^{2}\right)^{2} \leq \lambda^{2} \\
& \text { Simila }
\end{aligned}
$$

\section{ILLUSTRATION}

In this section, our approach is illustrated on the weighting transfer function generation for $H_{\infty}$ control of PLL network, introduced in [17]. A PLL (Phase-Locked Loop), used in micro-Electronics, is the feedback connection of an oscillator with a dynamical controller [3]. The synchronisation problem of these PLL can be formulated as a weighted- $H_{\infty}$ control problem. As noticed in [2], this requires the weighting function $W\left(T_{s}\right)$ to be expressed as an LFT in $T_{s}$.

The dynamics of the PLL in this example is given by:

$$
T_{s}(s)=\frac{b_{3} s^{3}+b_{2} s^{2}+b_{1} s+b_{0}}{a_{5} s^{5}+a_{4} s^{4}+a_{3} s^{3}+b_{2} s^{2}+b_{1} s+b_{0}}
$$


with $b_{0}=1.738 \times 10^{7}, b_{1}=6.172 \times 10^{6}, b_{2}=1.089 \times 10^{6}$, $b_{3}=2.178, a_{0}=1.738 \times 10^{7}, a_{1}=6.172 \times 10^{6}$, $a_{2}=1.093 \times 10^{6}, a_{3}=2.742 \times 10^{4}, a_{4}=238.6$ and $a_{5}=1 . T_{s}$ is lossy $\{x=-6, y=2, z=3.8\}$-dissipative.

TABLE I

ILLUSTRATIVE DISCRETE MASK

\begin{tabular}{llllll}
\hline Index & 1 & 2 & 3 & 4 & 5 \\
$\omega_{i}(\mathrm{rad} / \mathrm{s})$ & $1.0 \cdot 10^{-5}$ & $1.3 \cdot 10^{-4}$ & $1.7 \cdot 10^{-3}$ & 0.022 & 0.28 \\
$\left.W_{i}^{2}\right|_{d B}$ & 80 & 80 & 70 & 79 & 61 \\
\hline & 6 & 7 & 8 & 9 & 10 \\
& 3.6 & 46.4 & $6.0 \cdot 10^{2}$ & $7.7 \cdot 10^{3}$ & $1.0 \cdot 10^{5}$ \\
& 29 & 7.5 & 0.12 & $7.2 \cdot 10^{-4}$ & $4.3 \cdot 10^{-6}$ \\
\hline
\end{tabular}

The spectral mask of interest is discrete, is given in Table I and represented on Fig. 2 by red circles. $\mathcal{B}_{B}\left(T_{s}\right)$ is chosen as in (9) with $\left[\begin{array}{l|l}A_{B} & B_{B} \\ \hline C_{B} & D_{B}\end{array}\right]=\left[\begin{array}{ll|l}0 & 1 & 0 \\ 0 & 0 & 1 \\ \hline 0 & 0 & 1 \\ 0 & 1 & 0 \\ 1 & 0 & 0\end{array}\right]$. The optimisation problem $(i)$ of Theorem 5 is first solved using the Robust Control toolbox of Matlab. Then, the left-hand sides of (10) and (11) are respectively factorised as $\left[\begin{array}{ll}C_{N} & D_{N}\end{array}\right]^{T}\left[\begin{array}{ll}C_{N} & D_{N}\end{array}\right]$ and $\left[\begin{array}{ll}C_{D} & D_{D}\end{array}\right]^{T}\left[\begin{array}{ll}C_{D} & D_{D}\end{array}\right]$, with $C_{N}=\left[\begin{array}{ll}-3.0315150 & 4.0207130\end{array}\right], D_{N}=-1.0000000$ $C_{D}=\left[\begin{array}{ll}-1.0187513 & 2.0153471\end{array}\right], D_{D}=-0.99659474$. $W_{N}\left(T_{s}\right)$ and $W_{D}\left(T_{s}\right)$ are then obtained as in (29) and (30). Finally, as explained in [17], the weighting function $W\left(T_{s}\right)=\frac{W_{N}(T s)}{W_{D}(T s)}$ can be expressed as in (3) with:

$$
\left[\begin{array}{c|c}
A_{W} & B_{W} \\
\hline C_{W} & D_{W}
\end{array}\right]=\left[\begin{array}{cc|c}
0 & 1.0000000 & 0 \\
-1.0222322 & 2.0222333 & -1.0034170 \\
\hline-2.0092828 & 1.9984797 & 1.0034170
\end{array}\right]
$$

The modulus of the frequency response of $W\left(T_{s}\right)$ is represented on Fig. 2 by the blue curve.

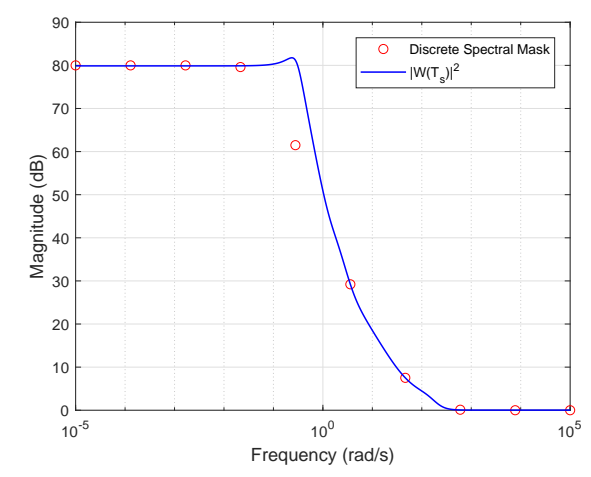

Fig. 2. $H_{\infty}$-Weights Generation Illustrative Example

\section{CONCLUSION}

In this paper, we have focused on the design of identical dissipative systems interconnection $W\left(T_{s}\right)$ under magnitude constraints. We have generalised the usual approach by independently extending each step from $T_{s}(s)=1 / s$ to any dissipative $T_{s}$, by especially generalising the spectral factorisation theorem (Theorem 3). While for lossless dissipative $T_{s}$ this enables to solve the design problem, the lossy part of general dissipative $T_{s}$ prevents from generalising the second step. We have finally revealed a new coupled approach able to convexly solve the design problems (Theorems 4 and 5).

Techniques generalised in this paper are used for numerous problems in Control and Signal Processing (refer to [16] for a sample of them) in the usual state-space approach. Future research directions include to apply these techniques to extended versions of these problems. Electronic system design, with new components of interest [18], is one of them.

\section{REFERENCES}

[1] P. Massioni and M. Verhaegen, "Distributed Control for Identical Dynamically Coupled Systems: A Decomposition Approach," IEEE Transactions on Automatic Control, vol. 54, no. 1, pp. 124-135, Jan 2009.

[2] M. Zarudniev, A. Korniienko, G. Scorletti, and P. Villard, "Network Internal Signal Feedback and Injection: Interconnection Matrix Redesign," in 52nd IEEE Conference on Decision and Control, Dec 2013.

[3] A. Korniienko, G. Scorletti, E. Colinet, and E. Blanco, "Performance Control for Interconnection of Identical Systems: Application to PLL network design," International Journal of Robust and Nonlinear Control, vol. 26, pp. 3-27, Jan 2016.

[4] S. Hara, H. Tanaka, and T. Iwasaki, "Stability Analysis of Systems With Generalized Frequency Variables," IEEE Transactions on Automatic Control, vol. 59, no. 2, pp. 313-326, Feb 2014.

[5] S. P. Boyd, L. El Ghaoui, E. Feron, and V. Balakrishnan, Linear Matrix Inequalities in System and Control Theory. SIAM, 1994, vol. 15.

[6] S. Hara, T. Iwasaki, and H. Tanaka, "Norm Computations for LTI Systems with Generalized Frequency Variables," in Proceedings of the 2010 American Control Conference, June 2010, pp. 1862-1867.

[7] G. Scorletti and L. E. Ghaoui, "Improved LMI Conditions for Gain Scheduling and Related Problems," Int. J. Robust and Nonlinear Control, vol. 8, no. 10, pp. 845-877, Aug. 1998.

[8] L. Rossignol, G. Scorletti, and V. Fromion, "Filter Design: a Finite Dimensional Convex Optimization Approach," International Journal of Robust and Nonlinear Control, vol. 13, no. 14, pp. 1317-1335, 2003.

[9] K. Zhou, J. Doyle, and K. Glover, Robust and Optimal Control. New Jersey: Prentice Hall, 1996.

[10] J. C. Willems, "Dissipative Dynamical Systems. II. Linear Systems with Quadratic Supply Rates," Arch. Ration. Mech. Anal, vol. 45, no. 5, pp. 352-393, 1972.

[11] T. W. Parks and C. S. Burrus, Digital Filter Design. WileyInterscience, 1987.

[12] S.-P. Wu, S. Boyd, and L. Vandenberghe, FIR Filter Design via Spectral Factorization and Convex Optimization. Boston, MA: Birkhäuser Boston, 1999, pp. 215-245.

[13] T. Iwasaki and S. Hara, "Generalized KYP Lemma: Unified Frequency Domain Inequalities with Design Applications," IEEE Transactions on Automatic Control, vol. 50, no. 1, pp. 41-59, 2005.

[14] R. A. Horn and C. R. Johnson, Matrix Analysis, 2nd ed. Cambridge University Press, 2012.

[15] J. Willems, "Least Squares Stationary Optimal Control and the Algebraic Riccati Equation," IEEE Transactions on Automatic Control, vol. 16 , no. 6, pp. 621-634, 1971.

[16] H. L. Trentelman and J. C. Willems, "The Dissipation Inequality and the Algebraic Riccati Equation," in The Riccati Equation. Berlin, Heidelberg: Springer Berlin Heidelberg, 1991.

[17] M. Zarudniev, "Synthèse de Fréquence par Couplage d'Oscillateurs Spintroniques," Ph.D. dissertation, Ecole Centrale de Lyon, Lyon, 2013, (In French). [Online]. Available: https://tel.archivesouvertes.fr/tel-00804561/document

[18] R. Weigel, D. P. Morgan, J. M. Owens, A. Ballato, K. M. Lakin, K. Hashimoto, and C. C. W. Ruppel, "Microwave Acoustic Materials, Devices, and Applications," IEEE Transactions on Microwave Theory and Techniques, vol. 50, no. 3, pp. 738-749, Mar 2002. 